\title{
APLICAÇÃO DOS PRINCÍPIOS DO PREHOSPITAL TRAUMA LIFE SUPPORT
}

\author{
Dayana Cristina Moraes ${ }^{1}$, Christiane Brey $^{2}$, Aline Cecilia Pizzolato ${ }^{3}$, Cristiano Caveião ${ }^{4}$, Leila Maria Mansano \\ Sarquis $^{5}$
}

RESUMO: Objetivou-se analisar a aplicação dos princípios do Prehospital Trauma Life Support pelas equipes do serviço pré-hospitalar móvel. Estudo observacional com 100 processos de atendimento às vítimas de trauma durante a admissão em um pronto socorro, em abril de 2014. Houve prevalência de vítimas de trauma entre 12 a 39 anos (64\%) e predomínio do sexo masculino (69\%). As equipes aplicaram os princípios do Prehospital Trauma Life Support na maioria das vítimas observadas, no entanto, identificou-se que $17 \%$ não estavam com a coluna cervical e vertebral imobilizadas, o corpo permaneceu imobilizado com cintos de fixação em apenas $31 \%$ dos casos, lesões não estavam protegidas em $15 \%$ das vítimas e $63 \%$ não foram despidas à procura de lesões. Ressalta-se que a aplicação dos princípios pelas equipes do serviço pré-hospitalar é primordial para padronizar ações, por meio de uma sequência lógica de atendimento universal. Sugere-se a capacitação continuada para essas equipes com base no Prehospital Trauma Life Support.

DESCRITORES: Atendimento Pré-Hospitalar; Trauma; Emergências; Capacitação em serviço; Enfermagem.

\section{APPLICATION OF PREHOSPITAL TRAUMA LIFE SUPPORT PRINCIPLES}

\begin{abstract}
The objective was to analyze the application of the Prehospital Trauma Life Support principles by the emergency medical teams. Observational study involving 100 care files of trauma victims during admission to an emergency medical service in April 2014. The prevalent cases of trauma victims were between 12 and 39 years of age (64\%), with a predominance of male victims (69\%). The teams applied the principles of Prehospital Trauma Life Support in most victims observed, but it was identified that $17 \%$ did not have their cervical and vertebral spine immobilized, the body remained immobilized using fixation belts in only $31 \%$ of the cases, injuries were unprotected in $15 \%$ of the victims and $63 \%$ were not undressed in search of injuries. It is highlighted that the emergency medical teams' application of the principles is fundamental to standardize actions through a logical sequence of universal care. Ongoing training is suggested for these teams, based on Prehospital Trauma Life Support.
\end{abstract}

DESCRIPTORS: Emergency Medical Services; Trauma; Emergencies; Inservice training; Nursing.

\section{APLICACIÓN DE LOS PRINCIPIOS DEL PREHOSPITAL TRAUMA LIFE SUPPORT}

RESUMEN: Fue objetivo del estudio analizar la aplicación de los principios del Prehospital Trauma Life Support por los equipos de servicio pre hospitalar móvil. Estudio observacional con 100 procesos de atendimiento a las víctimas de trauma durante la admisión en la emergencia, en abril de 2014. Fueron predominantes las víctimas de trauma entre 12 y 39 años (64\%) del sexo masculino (69\%). Los equipos aplicaron los principios del Prehospital Trauma Life Support en la mayoría de las víctimas observadas, sin embargo, se identificó que $17 \%$ no estaban con la columna cervical y vertebral inmovilizadas; el cuerpo permaneció inmovilizado con cintos de fijación en solamente $31 \%$ de los casos, lesiones no estaban protegidas en $15 \%$ de las víctimas y $63 \%$ no fueron desnudadas en la búsqueda de lesiones. Se destaca que la aplicación de los principios por los equipos del servicio pre hospitalar es primordial así como crear un patrón para las acciones, por medio de una secuencia lógica de atendimiento universal. Se sugiere la capacitación continuada para eses equipos con base en el Prehospital Trauma Life Support.

DESCRIPTORES: Atendimiento Pre Hospitalar; Trauma; Emergencias; Capacitación en servicio; Enfermería.

${ }^{1}$ Enfermeira. Mestranda em Enfermagem. Universidade Federal do Paraná. Curitiba, PR, Brasil.

${ }^{2}$ Enfermeira. Mestranda em Enfermagem. Docente do Centro Universitário Autônomo do Brasil-Unibrasil. Curitiba, PR, Brasil. ${ }^{3}$ Enfermeira. Doutoranda em Enfermagem. Universidade Federal do Paraná. Curitiba, PR, Brasil.

${ }^{4}$ Enfermeiro. Doutorando em Enfermagem. Docente do Centro Universitário Autônomo do Brasil-Unibrasil. Curitiba, PR, Brasil.

${ }^{5}$ Enfermeira. Pós-doutorada em Enfermagem. Docente da Universidade Federal do Paraná. Curitiba, PR, Brasil.

Autor Correspondente:

Recebido: 09/12/2015

Christiane Brey

Universidade Federal do Paraná

Finalizado: 01/06/2016

R. João Guerino Fabri, 438 - 82.940-170 - Curitiba, PR, Brasil

Email: christianebrey@gmail.com 


\section{- INTRODUÇÃO}

O trauma representa um problema de saúde pública de grande magnitude no Brasil, com profundas repercussões nas estruturas sociais, econômicas e políticas da sociedade ${ }^{(1)}$. Pode ser definido como um evento nocivo oriundo da transmissão de energia mecânica, química, térmica, elétrica ou por irradiação ${ }^{(2)}$. Trata-se de homicídios, suicídios, quedas, acidentes de trânsito, afogamentos, envenenamentos e vítimas de ferimento por arma branca e ferimento por arma de fogo ${ }^{(3)}$.

Nas últimas décadas, importantes avanços têm sido alcançados na área da saúde no Brasil pelo desenvolvimento de novas tecnologias. A era industrial, o aumento da velocidade dos veículos, as condições socioeconômicas e a própria condição da natureza humana são fatores que contribuíram para o crescimento progressivo das causas externas caracterizadas como traumatismos, lesões ou quaisquer outros agravos à saúde - intencionais ou não - de início súbito e como consequência imediata de violência ou outra causa exógena ${ }^{(4)}$.

As causas externas, desde o ano 2000, ocupam o terceiro lugar entre as causas de morte no Brasil(5). São a primeira causa de mortalidade na faixa etária de 1 a 39 anos e a maior parte da população atingida é do sexo masculino ${ }^{(1)}$.

Dados do Ministério da Saúde mostram que, em 2012, ocorreram aproximadamente 152 mil óbitos por causas externas. Foram notificados pelo Sistema de Informação de Mortalidade eventos relacionados com transportes terrestres, homicídios, agressões, suicídios, lesões autoprovocadas intencionalmente, quedas, queimaduras, afogamentos e eventos cuja intenção é considerada como indeterminada ${ }^{(6)}$.

Essa realidade aumenta a demanda de atendimento pré-hospitalar imediato às vítimas no local da ocorrência, com agilidade dos socorristas na abordagem inicial à vítima para identificação e estabilização de lesões. E, ainda, a necessidade de transporte apropriado e equipado para que a continuidade do atendimento à vítima, no ambiente intra-hospitalar de emergência seja eficiente e seguro às vítimas. Leva-se em consideração que os serviços de atendimento pré-hospitalar têm como objetivo a intervenção precoce a fim de reduzir os índices de morbimortalidade, enquanto que o atendimento intra-hospitalar promove medidas terapêuticas de suporte à vida ${ }^{(1)}$.

Nesse sentido, destaca-se um programa norte-americano, conhecido mundialmente como Prehospital Trauma Life Support - PHTLS (Atendimento Pré-Hospitalar ao Traumatizado), criado em 1981, que regulamenta o padrão no atendimento ao traumatizado em todo o mundo. Os conhecimentos necessários para diminuir a mortalidade estão nele contidos. Por conseguinte, a partir dele o socorrista garante decisões corretas no atendimento às vítimas com base em conhecimentos sólidos e científicos ${ }^{(2)}$.

Para a execução deste programa, deve-se seguir o Protocolo do ABCDE, considerado como um método mnemônico para atendimento primário do trauma. Ele tem base nos Estados Unidos da América, criado pelo Colégio Americano de Cirurgiões ${ }^{(2)}$ e visa melhorar o atendimento ao paciente traumatizado ao detectar precocemente as alterações fisiológicas que o colocam em risco de morte e/ ou sequelas.

A abordagem primária (ou ABCDE primário) tem como objetivo estabilizar os sinais vitais e identificar lesões que comprometam a vida da vítima através dos seguintes métodos: avaliação rápida e estabilização da via aérea (Airway), respiração (Breathing), circulação (Circulation), avaliação da condição neurológica (Disability) e exposição da pele à procura de lesões com manutenção da normotermia (Exposure and Enviromental control) ${ }^{(7)}$.

Os serviços de atenção pré-hospitalar necessitam adaptar-se ao aumento exponencial do número de vítimas de trauma. Para tanto, a aplicação de ações padronizadas permite garantir a qualidade no atendimento prestado a fim de reduzir os índices de mortalidade e minimizar sequelas. Sendo assim, os socorristas devem estar preparados e capacitados para tomada de decisões durante as situações de emergência.

Na mesma ótica, o Brasil aprovou uma linha de cuidado ao trauma por meio da Portaria $\mathrm{n}^{\circ} 1.366$ de 8 julho de $2013^{(8)}$, a qual preconiza a redução da morbidade e mortalidade, por meio da universalização 
e padronização de um modelo de atendimento ao paciente vítima de trauma com base na Sociedade Brasileira de Atendimento ao Traumatizado(1).

No âmbito da Enfermagem, a Lei do Exercício Profissional $n^{\circ} 7.498 / 86^{(9)}$, o Código de Ética dos Profissionais de Enfermagem ${ }^{(10)}$ e a Resolução $n^{\circ}$ 375/2011 do Conselho Federal de Enfermagem ${ }^{(11)}$ dispõem sobre a presença do enfermeiro no atendimento pré-hospitalar, na estruturação dos serviços, na prestação da assistência de enfermagem, na supervisão e coordenação da equipe de enfermagem (constituída pelos Técnicos de Enfermagem e Auxiliares de Enfermagem) e no desenvolvimento e gerenciamento de ações educativas. Assim sendo, compete ao enfermeiro conhecer a assistência prestada a fim de qualificar o atendimento oferecido.

Neste contexto, o objetivo do estudo foi analisar a aplicação dos princípios do PHTLS pelas equipes do serviço pré-hospitalar móvel.

\section{MÉTODO}

Estudo observacional, cujo foco é a investigação sem nenhuma intervenção ${ }^{(12)}$. O período de coleta de dados ocorreu entre os dias 05 e 30 de abril de 2014 em horários alternados, inclusive finais de semanas e feriados.

A pesquisa foi realizada em uma unidade de Pronto Socorro de um hospital público referência em trauma na cidade de Curitiba, estado do Paraná (PR). Foi observada a admissão de vítimas de trauma no Pronto Socorro, com o intuito de analisar a aplicação dos princípios do PHTLS pelo serviço préhospitalar, além de registrar dados sociodemográficos, como idade e sexo, empregando um roteiro de observação elaborado pelos pesquisadores e embasado no Protocolo ABCDE primário do trauma.

A opção em realizar o estudo de observação de um processo pré-hospitalar em um ambiente intrahospitalar se justifica pela lógica de que as etapas de manejo do atendimento pré-hospitalar à vítima de trauma se completam ao chegar ao hospital. Logo, o momento mais adequado para coleta dos dados seria durante a admissão no pronto socorro; além disso, as limitações para realizar o estudo de observação nas unidades móveis de urgência, por apresentarem um espaço físico reduzido e limitado aos socorristas em seu interior e pelos diversos pontos de base das ambulâncias, espalhados pela cidade de Curitiba.

Seguiu-se como critérios de inclusão: atendimento à vítima de trauma de qualquer natureza e idade, prestado por serviços especializados de atendimento pré-hospitalar como o Serviço Integrado de Atendimento ao Trauma em Emergência (SIATE), Serviço de Atendimento Móvel de Urgência (SAMU) e outras ambulâncias e helicópteros de resgate, durante a entrada na unidade hospitalar fixa de emergência.

Utilizando dados de domínio público, a população do estudo foi composta por 500 processos de atendimento a vítimas de trauma na instituição, sendo este um número médio de atendimentos a cada mês.

Para a seleção da amostra foi utilizado o plano amostral, embasado no processo de Amostragem das Proporções, devido à grande quantidade de variáveis a serem investigadas na pesquisa de acordo com o Protocolo ABCDE do trauma.

Ficou estabelecida a população com $\mathrm{N}=500$ e fixado o nível de confiança da amostra em 95\% e erro máximo de $7 \%$. Também ficou calculado o quantitativo da amostra para o total de processos de atendimento, incluído um adicional de $20 \%$ com a previsão de possíveis dificuldades durante a coleta de dados; assim, $\mathrm{N}=100$ processos de atendimento a vítimas de trauma.

A avaliação do processo de atendimento, quanto à imobilização e aos procedimentos realizados pelo serviço pré-hospitalar, seguiu as ações recomendadas pelo programa PHTLS e o Protocolo ABCDE do trauma. Os dados foram analisados de maneira estatística descritiva, em planilha eletrônica (Microsoft Office $\mathrm{Exce}^{\circledR}$ ) e os resultados são apresentados na forma de frequências simples e absolutas. A pesquisa foi realizada após aprovação institucional, em 02/04/2014, e do Comitê de Ética em Pesquisa (CAAE n 25686313.2.0000.0095). 
Entre as 100 vítimas admitidas, houve a prevalência de indivíduos dentro da faixa etária de 12 a 39 anos com percentual de $64 \%$ (64), e predomínio do sexo masculino, totalizando $69 \%$ (69) de homens atendidos.

Os resultados apresentam as percentagens resultantes do processo de atendimento na admissão de vítimas de trauma no Pronto Socorro com relação ao manejo do ABCDE primário, bem como o intervalo de confiança (IC=95\%). Conforme ilustrado na Tabela 1, o manejo da etapa A - Vias aéreas e controle da coluna cervical, 99\% (99) dos atendimentos observados assegurava a permeabilidade das vias aéreas; entretanto, este dado corresponde ao maior número de vítimas conscientes admitidas no pronto socorro no período pesquisado e reflete no percentual de 99\% (99) de não aplicação da cânula orofaríngea. Não havia indicação de abordagem avançada da via aérea em 97\% (97) das vítimas; para 3\% (03), apesar do indicativo, não garantia a permeabilidade por meio de via aérea definitiva.

Tabela 1 - Distribuição do processo de atendimento observado em relação ao manejo da etapa A - vias aéreas e controle da coluna cervical. $\mathrm{N}=100$. Curitiba, PR, Brasil, 2014

\begin{tabular}{|c|c|c|}
\hline Manejo da etapa A & n (\%) & $I C=95 \%$ \\
\hline \multicolumn{3}{|c|}{ Estava assegurada a permeabilidade das vias aéreas? } \\
\hline Sim & $99(99)$ & $(97 ; 100)$ \\
\hline Não & $1(1)$ & $(0 ; 2,95)$ \\
\hline Não se aplica & $0(0)$ & $(0 ; 0)$ \\
\hline \multicolumn{3}{|c|}{ A vítima permaneceu com cânula orofaríngea? } \\
\hline Sim & 1(1) & $(0 ; 2,95)$ \\
\hline Não & $0(0)$ & $(0 ; 0)$ \\
\hline Não se aplica & $99(99)$ & $(97 ; 100)$ \\
\hline \multicolumn{3}{|c|}{ Havia indicação para técnica de manejo avançado da via aérea? } \\
\hline Sim & $3(3)$ & $(0 ; 6,3)$ \\
\hline Não & $97(97)$ & $(93,6 ; 100,3)$ \\
\hline Não se aplica & $0(0)$ & $(0 ; 0)$ \\
\hline \multicolumn{3}{|c|}{ Admitida em tábua de imobilização dorsal? } \\
\hline Sim & $83(83)$ & $(75,6 ; 90,3)$ \\
\hline Não & $17(17)$ & $(9,6 ; 24,3)$ \\
\hline Não se aplica & $0(0)$ & $(0 ; 0)$ \\
\hline \multicolumn{3}{|c|}{ Permaneceu com colar cervical? } \\
\hline Sim & $80(80)$ & $(72,1 ; 87,8)$ \\
\hline Não & $20(20)$ & $(12,1 ; 27,8)$ \\
\hline Não se aplica & $0(0)$ & $(0 ; 0)$ \\
\hline \multicolumn{3}{|c|}{ A cabeça estava imobilizada de modo a não permitir qualquer movimento? } \\
\hline Sim & $34(34)$ & $(24,1 ; 43,2)$ \\
\hline Não & $66(66)$ & $(56,7 ; 75,2)$ \\
\hline Não se aplica & $0(0)$ & $(0 ; 0)$ \\
\hline \multicolumn{3}{|c|}{ O corpo estava imobilizado de modo a não permitir qualquer movimento? } \\
\hline Sim & $31(31)$ & $(24,7 ; 43,2)$ \\
\hline Não & $69(69)$ & $(56,7 ; 75,2)$ \\
\hline Não se aplica & $0(0)$ & $(0 ; 0)$ \\
\hline
\end{tabular}


Quanto ao controle da coluna cervical que integra a etapa A: 83\% (83) das vítimas foram admitidas em prancha longa. Entretanto, esse elevado percentual não deve ser sinônimo de acomodação, haja vista que em $17 \%$ (17) das vítimas não havia controle da coluna vertebral. O colar cervical também tem como finalidade a proteção, 80\% (80) dos traumatismos entraram com o dispositivo, mas 20\% (20) estavam sem colar. Ainda neste contexto, a cabeça não estava totalmente imobilizada com imobilizador lateral de cabeça em $66 \%$ (66) dos atendimentos. O corpo permaneceu imobilizado com cintos de fixação em apenas 31\% (31) dos casos.

O manejo da etapa B - Respiração (ventilação), apresentava um total de 92\% (92) de vítimas com saturação de oxigênio maior que 95\% (95). Entretanto, uma vítima com saturação de oxigênio menor que $95 \%$ (95) foi admitida no pronto socorro sem oxigênio suplementar.

Em relação aos resultados identificados na etapa C - Circulação (sangramento), percebeu-se que $87 \%$ (87) das vítimas observadas não precisavam de aplicação de controle de hemorragias. Com relação às vítimas que apresentavam lesões foram 47\% (47), observou-se que não estavam protegidas em 15\% (15) das vítimas. Não foi observada a manutenção de dois acessos venosos calibrosos em $82 \%$ (82) dos traumatismos. A reposição volêmica teve o Ringer Lactato como volume utilizado em 16\% (16) dos atendimentos. A imobilização de prováveis fraturas ocorreu em 19\% (19) dos casos.

Durante os processos de atendimento em relação à etapa D - Incapacidade (avaliação neurológica) a Escala de Coma de Glasgow (ECG) foi utilizada em 100\% (100), enquanto a avaliação das alterações pupilares foi de $89 \%$ (89), dado obtido mediante a observação do preenchimento das fichas de atendimento pelo serviço pré-hospitalar ou pelo repasse destas informações na unidade fixa de emergência.

O manejo da etapa E - Exposição/Ambiente, apontava que 63\% (63) das vítimas não foram despidas à procura de lesões e 67\% (67) dos pacientes não estavam aquecidos com auxílio de cobertor ou manta térmica.

\section{DISCUSSÃO}

A análise desta pesquisa ocorreu sob o olhar do protocolo PHTLS, que há anos qualifica o atendimento ao traumatizado em mais de 60 países. Além disso, no Brasil a Sociedade Brasileira de Atendimento ao Traumatizado norteia os princípios do atendimento pré-hospitalar por meio do ABCDE do trauma.

Esta pesquisa permitiu identificar que, durante o processo de atendimento, as vítimas de trauma admitidas no Pronto Socorro foram predominantemente do sexo masculino. Este perfil é semelhante a outros estudos que indicam pessoas do sexo masculino como vítimas mais frequentes de trauma ${ }^{(1,13)}$, sendo consequência provável da maior exposição masculina no trânsito e por comportamentos determinados socioculturais, em que os homens assumem maiores situações de risco ${ }^{(13)}$. Quanto ao perfil determinado pela idade, houve maior incidência entre a faixa etária de 12 a 39 anos, dado que se contrapões em parte aos de outros estudos, os quais apontam maior ocorrência de trauma em vítimas entre 20 a $39 \operatorname{anos}^{(1,4,13)}$. A partir desse perfil das vítimas, o serviço de atendimento de emergência poderá desenvolver estratégias que visem um atendimento padronizado e de qualidade.

Em análise, os achados da presente pesquisa retratam peculiaridades no processo de atendimento às vítimas, as quais apontam necessidade de intervenção. A avaliação e o atendimento inicial do traumatizado requerem uma abordagem sistemática, por meio das cinco etapas contidas no exame primário, caracterizados como ABCDE do trauma.

A primeira etapa (A) do método visa garantir a permeabilidade das vias aéreas por meio de manobras manuais de abertura, como tração da mandíbula ou elevação do mento ou por meios mecânicos: cânula orofaríngea, em pacientes inconscientes em decorrência da queda da língua, cânula nasofaríngea ou intubação endotraqueal. Pacientes com rebaixamento do nível de consciência, ou com Escala de Coma de Glasgow (ECG) igual ou menor que oito, exigem uma via aérea definitiva ${ }^{(2)}$. Nesta etapa é indicado o controle de cervical.

A investigação revela que $1 \%$ das vítimas não tinha assegurada a permeabilidade das vias aéreas. Diante dessa realidade, o socorrista deve ser capaz de identificar a necessidade de intervenção avançada 
no local da ocorrência e promover meios de suporte básico de vida até a chegada de apoio, com o objetivo de evitar sequelas irreparáveis como consequência da hipóxia. Afinal, a perda de uma via aérea mata mais rapidamente do que a perda da capacidade de respiração, que mata mais rapidamente do que a redução do volume sanguíneo circulante ${ }^{(14)}$.

E com relação ao controle de cervical, os profissionais atuantes no atendimento pré-hospitalar móvel precisam reconhecer os riscos relacionados à não imobilização da coluna. As lesões instáveis da coluna vertebral sem lesão neurológica, principalmente em pacientes politraumatizados, acarretam grande potencial de lesão adicional das estruturas nervosas durante o resgate e transporte dos pacientes. Logo, as vítimas devem ser imobilizadas em posição alinhada e neutra, para impedir que qualquer movimento de coluna possa resultar em lesão de medula ${ }^{(15)}$.

Neste contexto, torna-se necessário manter o uso dos dispositivos de imobilização cervical e toracolombar por meio de colar cervical, coxins laterais, imobilizadores de cabeça, cintos de fixação e prancha rígida ${ }^{(2,16)}$. Alguns autores reiteram que o diagnóstico desse tipo de lesão não é prioritário, mas a proteção é essencial ${ }^{(16)}$.

Por conseguinte, esses dispositivos devem ser mantidos até a exclusão de fratura ou lesão de ligamentos da coluna ${ }^{(1-2)}$. O transporte em prancha rígida é fundamental até a realização do exame clínico e do estudo radiológico, se necessário. No ambiente hospitalar, o colar cervical de vítimas conscientes e orientadas, sem trauma cervical, sem dor à palpação e à mobilização passiva, pode ser retirado pelo médico, na própria sala de emergência, sem a necessidade de realização de uma radiografia complementar ${ }^{(1)}$.

Destacam-se nesta pesquisa os dados observados como fator preocupante em relação ao controle da coluna vertebral, uma vez que as vítimas de trauma admitidas sem tábua de imobilização dorsal totalizaram $17 \%$, sem colar cervical perfizeram $20 \%$ dos atendimentos; e, por fim, a falha na fixação da cabeça e a do corpo, de modo a não permitir qualquer movimento, ocorreu em $66 \%$ e $69 \%$ dos casos, respectivamente.

Em relação à manutenção da respiração, etapa $\mathrm{B}$ do método mnemônico, aponta-se o percentual de $1 \%$ de vítimas admitidas com saturação menor que $95 \%$ sem oxigênio suplementar, os dados estão em consonância com os riscos recorrentes da hipóxia. A oferta de oxigênio aos tecidos é um dos objetivos do atendimento inicial, pois todo paciente traumatizado deve receber oxigênio suplementar com fluxo de 10L/minuto através de máscara com reservatório, com a finalidade de manter a oximetria de pulso maior que $95 \%{ }^{(1-2)}$.

A hipóxia é resultante da ventilação inadequada dos pulmões e falta de oxigenação dos tecidos, e ocasiona o acúmulo de dióxido de carbono no organismo, o que leva à acidose e eventualmente à morte, caso não haja tratamento adequado ${ }^{(2)}$. Logo, o socorrista deve avaliar a função ventilatória e a capacidade da vítima em manter a oxigenação de forma efetiva.

Com relação à etapa $\mathrm{C}$ do método mnemônico, os dados evidenciados sobre o controle das hemorragias são satisfatórios. No entanto, os aspectos analisados referentes à proteção das lesões, demonstraram que em $15 \%$ dos casos observados, as lesões não estavam protegidas. Verificou-se também ausência de dois acessos venosos calibrosos em $82 \%$ das vítimas, a não utilização do Ringer Lactato em $84 \%$ das reposições volêmicas e o percentual de $2 \%$ para prováveis fraturas não imobilizadas.

No cenário do atendimento pré-hospitalar, reduzir a infecção por micro-organismos patogênicos pela proteção das lesões é fundamental. A principal causa de mortalidade tardia em vítimas de trauma no hospital é a infecção ${ }^{(17)}$.

Nessa ótica, a literatura descreve a hemorragia como a principal causa de mortes pós-traumáticas evitáveis, cujo agravo ocasiona graus variáveis de coagulopatia, hipotermia, acidose metabólica e ainda, hipovolemia e consequentemente hipotensão caso não ocorra a manutenção da pressão arterial necessária ${ }^{(18)}$. O controle da hemorragia externa é prioritário, pelo uso de compressão direta ou uso cauteloso de torniquetes ${ }^{(2)}$.

O Ringer Lactato é o fluido de escolha para iniciar a reposição volêmica; imediatamente após a administração, o cristaloide enche o território vascular depletado pela perda de sangue, o que melhora 
a pré-carga e o débito cardíaco. O Soro Fisiológico a 0,9\% é uma alternativa aceitável embora possa ocorrer hipocloremia ${ }^{(1-2)}$.

No entanto, essa conduta clássica na ressuscitação volêmica com infusão de grandes volumes de fluidos tem sido motivo de controvérsia, pois existem evidências de que as estratégias convencionais podem exacerbar a coagulopatia, o sangramento e a morbimortalidade ${ }^{(18)}$.

Trabalhos experimentais mostram que a administração agressiva de cristaloides na presença de hemorragia não controlada promove a continuação do sangramento e aumenta a mortalidade. Por outro lado, a diminuição da quantidade de fluidos pode levar à hipoperfusão tecidual, falência orgânica e óbito, mais do que controle da hemorragia. É aconselhável e apropriada a manutenção de uma pressão arterial média entre 60 e $80 \mathrm{mmHg}$ de acordo com a literatura ${ }^{(19)}$.

Acerca do elevado percentual de vítimas admitidas sem acesso venoso periférico no Pronto Socorro de observação desta pesquisa, pode estar relacionado com o elevado número de atendimentos executados por profissionais (como os bombeiros militares) que realizam suporte básico de vida, com ações não invasivas no uso de suas atribuições legais, conforme a Portaria $n^{\circ} 2048$ de $2002^{(20)}$, do Ministério da Saúde, que regulamenta a legislação sobre unidades móveis.

As lesões decorrentes de eventos traumáticos resultam em deficiências e incapacidades temporárias ou permanentes, as quais podem comprometer a capacidade para execução das atividades diárias, bem como a qualidade de vida. Nessas situações, as lesões frequentes são as fraturas, as contusões e as luxações, que necessitam de longos períodos de recuperação, com importantes custos econômicos e sociais $^{(17)}$. Por isso, cabe ao socorrista imobilizar as prováveis fraturas com o objetivo de evitar agravos.

Com relação à etapa $\mathrm{D}$ do método mnemônico, evidencia-se o manejo da avaliação neurológica em 100\% dos atendimentos pela aplicação da Escala de Coma de Glasgow e $89 \%$ pela avaliação das pupilas. Ambos são métodos rápidos e práticos que podem ser utilizados diversas vezes, o que favorece a aplicação e justifica os resultados. Afinal, caracterizar a resposta neurológica no momento do atendimento é essencial para estabelecer o nível de consciência da vítima, com a aplicação da Escala de Coma de Glasgow, assim como o tamanho, simetria e reação das pupilas.

Um quadro de rebaixamento do nível de consciência pode representar a diminuição na oxigenação ou perfusão cerebral, ou ainda ser resultado do trauma direto ao cérebro. Imediatamente, há necessidade de reavaliação da ventilação, oxigenação e perfusão. De modo geral, toda alteração do nível de consciência deve ser considerada como etiologia de um traumatismo cranioencefálico, exceto o uso de álcool e drogas ${ }^{(21)}$.

Quanto às alterações nas pupilas, estas podem estar relacionadas não apenas a condições patológicas, mas ao uso de substâncias alcoólicas e drogas e por estado psicológico alterado ${ }^{(21)}$.

Posteriormente à avaliação neurológica, deve-se proceder à avaliação da etapa $\mathrm{E}$ do método mnemônico, por meio da exposição da vítima quando em ambiente interno, com o objetivo de efetuar o exame físico. Como também, nesta etapa, o controle da hipotermia com o uso de cobertores térmicos que minimizam a perda de calor ${ }^{(19)}$.

A hipotermia interfere em diversas funções fisiológicas como metabolismo basal, transporte de oxigênio e gás carbônico, concentrações de íons hidrogênio no sangue, no sistema nervoso, cardiovascular, respiratório, urinário, digestivo e nas alterações hematológicas, hídricas, eletrolíticas e hormonais ${ }^{(17)}$.

Em última análise, é preciso detectar e controlar riscos precocemente, a fim de evitar danos irreparáveis como consequência das lesões medulares, da hipóxia, da hipovolemia, da hipotermia e outros agravantes decorrentes do atendimento inadequado, com o objetivo de melhorar o prognóstico das vítimas de trauma.

Por fim, entre as limitações da pesquisa encontra-se o tamanho amostral, pois a heterogeneidade da população (não prevista anteriormente aos resultados) gera viés em erros amostrais maiores que $5 \%$. Embora limitada, a pesquisa produziu conhecimento a respeito de um problema de saúde pública, frequente durante o processo de atendimento a vítimas de trauma, o que já é motivação suficiente para outros estudos que possam contribuir para melhoria do atendimento pré-hospitalar. 
O manejo constante de vítimas pelos profissionais atuantes no atendimento pré-hospitalar móvel não justifica a exclusão dos cuidados quanto ao atendimento correto - ao contrário, a qualidade deve ser ampliada por meio da prática diária. Para tanto, os serviços de atenção pré-hospitalar móvel devem estar preparados para as mais diversas situações de urgência e emergência, e sua prática deve estar calcada em conhecimento teórico.

Sugere-se que os serviços de atendimento pré-hospitalar ao trauma que fizeram parte dessa pesquisa unifiquem a assistência com o uso de uma linguagem universal. E, que seja executada com base em uma sequência lógica de atendimento, focada nas prioridades das vítimas.

Tal pesquisa recomenda, ainda, que educação em serviço e treinamentos constantes sejam oferecidos a todos que fazem parte do processo, para que as condutas sejam padronizadas com vistas à atuação correta, segundo preconizado na literatura, nos programas e protocolos.

Para tanto, a utilização dos princípios do PHTLS e capacitações constantes, fornecem subsídios para a padronização da assistência dentro dos serviços pré-hospitalares móveis. De modo a refletir diretamente na qualidade do atendimento à vítima, por uma assistência individualizada, efetiva, segura, minimizando sequelas e agravos.

\section{REFERÊNCIAS}

1. Ministério da Saúde (BR). Secretaria de Atenção à Saúde. Departamento de Atenção Especializada. Manual instrutivo da Rede de Atenção às Urgências e Emergências no Sistema Único de Saúde (SUS). [Internet] 2013 [acesso em 30 ago 2014]. Disponível: http://bvsms.saude.gov.br/bvs/publicacoes/manual_instrutivo_rede_ atencao_urgencias.pdf.

2. PHTLS - Prehospital trauma life support. Atendimento pré-hospitalar ao traumatizado: básico e avançado. $7^{\mathrm{a}}$ ed. Rio de Janeiro: Elsevier; 2012.

3. Falck AC, Alm A, Lindstrom V. Has increased nursing competence in the ambulance services impacted on prehospital assessment and interventions in severe traumatic brain-injured patients? Scand J Trauma Resusc Emerg Med. [Internet] 2014; 22(20) [acesso em 20 ago 2015]. Disponível: http://dx.doi.org/10.1186/1757-7241-22-20.

4. Gonsaga RAT, Rimoli CF, Pires EA, Zogheib FS, Fujino MVT, Cunha MB. Avaliação da mortalidade por causas externas. Rev. Col. Bras. Cir. [Internet] 2012; 39(4) [acesso em 10 set 2015]. Disponível: http://dx.doi.org/10.1590/ S0100-69912012000400004

5. Ministério da Saúde (BR). Informações de Saúde. Mortalidade Proporcional por grupo de causas. [Internet] 2010 [acesso em 10 mar 2015]. Disponível: http://tabnet.datasus.gov.br/cgi/deftohtm.exe?idb2010/c04.def.

6. Ministério da Saúde (BR). Informações de saúde. Evolução da Mortalidade por Violência no Brasil e regiões. [Internet] 2012 [acesso em 24 mai 2014]. Disponível: http://tabnet.datasus.gov.br/cgi/deftohtm.exe?idb2012/c09. def.

7. Cestari VRF, Sampaio LRL, Barbosa IV, Studart RMB, Moura BBF, Araujo ARC. Tecnologias do cuidado utilizadas pela enfermagem na assistência ao paciente politraumatizado: revisão integrativa. Cogitare Enferm. [Internet] 2015; 20(4) [acesso em 09 jan 2015]. Disponível: http://dx.doi.org/10.5380/ce.v20i4.40819.

8. Ministério da Saúde (BR). Portaria $n^{\circ}$ 1.366, de 8 de julho de 2013. Estabelece a organização dos Centros de Trauma, estabelecimentos de saúde integrantes da Linha de Cuidado ao Trauma da Rede de Atenção às Urgências e Emergências (RUE) no âmbito do Sistema Único de Saúde (SUS). [Internet] 2013 [acesso em 30 set 2014]. Disponível: http://bvsms.saude.gov.br/bvs/saudelegis/gm/2013/prt1366_08_07_2013.html.

9. Brasil. Lei n. 7.498, de 25 de junho de 1986. Dispõe sobre a regulamentação do Exercício da Enfermagem e dá outras providências. Diário Oficial da República Federativa do Brasil, Brasília, 26 jun. 1986. Seção 1:1.

10. Conselho Federal de Enfermagem. Resolução nº 311/2007. Aprova a Reformulação do Código de Ética dos 
Profissionais de Enfermagem. Rio de Janeiro: COFEN; 2007.

11. Conselho Federal de Enfermagem. Resolução $n^{\circ} 375 / 2011$. Dispõe sobre a presença do Enfermeiro no Atendimento Pré-Hospitalar e Inter-Hospitalar, em situações de risco conhecido ou desconhecido. Brasília: COFEN; 2011.

12. da Silva GA, Otta E. Revisão sistemática e meta-análise de estudos observacionais em Psicologia. Rev. Costarric. Psicol. [Internet] 2014; 33(2) [acesso em 23 ago 2015]. Disponível: http://rcps-cr.org/wp-content/themes/rcps/ descargas/2014/2/6-RCP-Vol.33-No2.pdf.

13. Cabral APS, de Souza WV, de Lima LMC. Serviço de Atendimento Móvel de Urgência: Um observatório dos acidentes de transportes terrestres em nível local. Rev. bras. epidemiol. [Internet] 2011; 14(1) [acesso em 08 jan 2016]. Disponível: http://dx.doi.org/10.1590/S1415-790X2011000100001.

14. Lampi M, Vikstrom T, Jonson CO. Triage performance of Swedish physicians using the ATLS algorithm in a simulates mass casualty incident: a prospective cross-sectional survey. Scand J Trauma Resusc Emerg Med. [Internet] 2013; 21(90) [acesso em 04 abr 2016]. Disponível: http://dx.doi.org/10.1186/1757-7241-21-90.

15. Schweitzer G, do Nascimento ERP, do Nascimento KC, Moreira AR, Bertoncello KCG. Protocolo de cuidados de enfermagem no ambiente aeroespacial à pacientes traumatizados: cuidados durante e após o voo. Texto Contexto Enferm. [Internet] 2011; 20(3) [acesso em 04 abr 2016]. Disponível: http://dx.doi.org/10.1590/S010407072011000300008 .

16. Del Rossi G, Rechtine GR, Conrad BP, Horodyski MB. Is Sub-occipital Padding Necessary to Maintain Optimal Alignment of the Unstable Spine in the Prehospital Setting?. J Emerg Med. [Internet] 2013; 45(3) [acesso em 20 set 2015] Disponível: http://dx.doi.org/10.1016/j.jemermed.2013.01.042.

17. Sallum AMC, de Sousa RMC. Diagnósticos de enfermagem em vítimas de trauma nas primeiras seis horas após o evento. Acta paul. enferm. [Internet] 2012; 25(2) [acesso em 08 jan 2016]. Disponível: http://dx.doi.org/10.1590/ S0103-21002012000200016.

18. Barbosa Neto JO, de Moraes MFB, Nani RS, Rocha Filho JA, Carmona MJC. Ressuscitação hemostática no choque hemorrágico traumático: relato de caso. Rev. Bras. Anestesiol. [Internet] 2013; 63(1) [acesso em 15 fev 2015]. Disponível: http://dx.doi.org/10.1590/S0034-70942013000100008.

19. Simões RL, Duarte Neto C, Maciel GSB, Furtado TP, Paulo DNS. Atendimento pré-hospitalar à múltiplas vítimas com trauma simulado. Rev. Col. Bras. Cir. [Internet] 2012; 39(3) [acesso em 25 ago 2015]. Disponível: http://dx.doi. org/10.1590/S0100-69912012000300013.

20. Ministério da Saúde (BR). Portaria GM/MS n. 2048/ GM, de 5 de Novembro de 2002. Dispõe sobre o Regulamento Técnico dos Sistemas Estaduais de Urgência e Emergência. Brasília: Ministério da Saúde; 2002.

21. Bertoncello KCG, Cavalcanti CDAK, Ilha P. Análise do perfil do paciente como vítima de múltiplos traumas. Cogitare Enferm. 2012; 17(4) [acesso em 06 abr 2016]. Disponível: http://dx.doi.org/10.5380/ce.v17i4.30380. 